\title{
Tuning random lasing in photonic glasses
}

\author{
Michele Gaio, ${ }^{1, *}$ Matilda Peruzzo, ${ }^{1}$ and Riccardo Sapienza ${ }^{1}$ \\ ${ }^{1}$ Department of Physics, King's College London, Strand, London WCR 2LS, United Kingdom.
}

compiled: June 26, 2021

\begin{abstract}
We present a detailed numerical investigation of the tunability of a diffusive random laser when Mie resonances are excited. We solve a multimode diffusion model and calculate multiple light scattering in presence of optical gain which includes dispersion in both scattering and gain, without any assumptions about the $\beta$ parameter. This allows us to investigate a realistic photonic glass made of latex spheres and rhodamine and to quantify both the lasing wavelength tunability range and the lasing threshold. Beyond what is expected by diffusive monochromatic models, the highest threshold is found when the competition between the lasing modes is strongest and not when the lasing wavelength is furthest from the maximum of the gain curve.
\end{abstract}

Random lasers (RL) are mirror-less lasing systems which have attracted a lot of interest due to their structural simplicity. Nowadays they have been studied in a vast variety of scattering systems ranging from semiconductor powder to biological tissue and biocompatible materials [1]. Random lasing originates from a complex out-of-equilibrium phenomenon with rich multimodes features [2] and surprising statistical features [3]. Despite its potential for practical applications [1], random lasing technology is still in its infancy with pioneering applications such as low coherence light source [4] and biosensing [5]. One of the factors that has limited practical applications is the difficulty of controlling the frequency and directionality of the emission. In conventional lasers the lasing emission can be tuned by engineering the high finesse cavity which provides the feedback and thus defines the lasing mode. Instead, feedback in RL is provided by multiple scattering and the lasing emission properties are determined by the complex interplay between gain and losses. Recent experiments have shown lasing emission controlled by exploiting scattering dispersion via resonant scattering sustained by spherical particles $[6,7]$ or by gain dispersion achieved by artificially increasing absorption in a spectral band [8]. Active tuning of the lasing properties has also been achieved by shaping the pump profile to selectively excite one or a few lasing modes [9-11].

Different theoretical approaches to model random lasing action have been developed which combine multiple scattering and gain. For uncorrelated random systems in which interference between the scattered waves can be neglected, diffusive models are very accurate even in presence of optical gain [12, 13] and they provide the time evolution of the lasing process and a smooth lasing spectrum with no spiking lasing behaviour [14]. The radiative transport model with gain can also be solved for

* Corresponding author: michele.gaio@kcl.ac.uk instance with Monte Carlo simulations which consider a random walk of photons $[15,16]$ and in which amplification of single paths can be important in defining the spectral properties [17], and by solving the complete radiative transfer equations [18]. These approaches allow the study of large systems ( $>100$ s mean free paths) and geometries similar to real experiments. Recently, more complete models including field calculations and interference effects have been developed, based for example on Maxwell-Bloch equations [19, 20] and the finitedifference time-domain solution of Maxwell equations in nonlinear media [21-23], but limited to volumes of a few wavelengths cubed.

In this letter, we investigate the range of tunability of a diffusive RL with intensity feedback. We solve the diffusion equations for typical experimental configurations by including the full spectral dispersion of both scattering and gain, beyond the stationary case [12] and few modes model [14], and we calculate the full spatial, spectral and lasing dynamics, including mode competition and threshold variation.

The relevant quantities in a diffusive model are the transport mean free path $\ell_{t}$, the diffusion constant $D=$ $\ell_{t} v / 3$, and $v$ the speed of energy in the medium, which can all be modulated by resonant scattering [24]. The optical gain is provided by organic molecules, rhodamine 6G in this case, described by the stimulated emission cross-section $\sigma_{e}$, the absorption cross section $\sigma_{a}$, and the lifetime of the excited state $\tau$. The molecules composing the gain can be brought to the excited state by an intense pump laser. Here we label $N_{1}$ the density of molecules in the excited state and $N$ the total molecule density. We model a translational invariant slab geometry similar to most experiments with the 1D diffusion equation for the pump $W_{p}(x, t)$ and the emitted light $W^{i}(x, t)$. These equations are coupled to a four level system gain described by one rate equation for the ra- 
diative transition. The set of equation is the following:

$$
\begin{aligned}
\frac{\partial N_{1}}{\partial t} & =\sigma_{a} W_{p}\left[N-N_{1}\right] v-\sum_{i} \sigma_{e}^{i} W^{i} N_{1}-\frac{N_{1}}{\tau} \\
\frac{\partial W_{p}}{\partial t} & =D \frac{\partial^{2} W_{p}}{\partial x^{2}}-\sigma_{a} W_{p}\left[N-N_{1}\right] v+\frac{I_{p}}{\ell_{e}} \\
\frac{\partial W^{i}}{\partial t} & =D \frac{\partial^{2} W_{i}}{\partial x^{2}}+\sigma_{e}^{i} W^{i} N_{1} v+\frac{\phi^{i}}{\tau} N_{1}
\end{aligned}
$$

where the emitted light is discretised in spectral bands $\lambda_{i}$, and $i=1 . . n$. $I_{p}(x, t)$ is the intensity temporal profile of the pump, $\ell_{e}$ is its extinction length and $v$ is the velocity of light in inside the medium. The spontaneous emission (fluorescence) spectrum defines the quantities $\phi^{i}$, with $\sum \phi^{i}=1$. When compared to previous works $[8,13]$, the spontaneous emission factor $\beta$ is not a free parameter any more, but comes as a solution to the problem. We solve the system of coupled differential equations (1-3) by means of standard ODE solvers implemented in MATLAB. The main advantage of this model is that it includes spectral dispersion of scattering, absorption and gain.

We choose to simulate typical experimental conditions [6]: the sample is a slab $50 \mu \mathrm{m}$ thick, with $\ell_{t}=1.5 \mu \mathrm{m}$, and doped with a concentration of $1 \mathrm{mM}$ of rhodamine 6G dye whose emission properties are taken from ref. [25]. The system is pumped with a 6 ns laser pulse at a wavelength of $532 \mathrm{~nm}$.

Firstly, we consider a conventional RL, such as a polydisperse $\mathrm{TiO}_{2}$ powder, where $\ell_{t}$ is not dispersive but roughly constant over the gain spectrum. In Fig. 1(a) we plot the peak spectral fluence diffusing from the system at the air-sample interface together with the full width half maximum (FWHM) of the emission spectra as a function of the pump energy density. The onset of the lasing emission is at $P=0.07 \mathrm{~mJ} / \mathrm{mm}^{2}$, at higher pump intensities the peak fluence increases super-linearly until gain saturation is reached; at the same time the emission width quickly decreases from the broad fluorescence emission to a narrow almost constant value at saturation. The threshold of the lasing emission is usually identified in analogy to conventional lasing by considering the change in slope of the pump-peak emission intensity relation or by considering the narrowing of the lasing emission. Throughout this letter we identify the threshold as the half narrowing of the emission spectrum, which is a typical experimental parameter more accessible than the change of slope [26]. The FWHM of the emission narrows from the initial $\sim 35 \mathrm{~nm}$ of the rhodamine fluorescence spectrum to $\sim 0.8 \mathrm{~nm}$ of maximum lasing narrowing. The $\beta$ parameter can be calculated from Fig. 1(a) and is $\beta \simeq 0.04$. In Fig. 1(b) the emission spectra below and above threshold and for an intermediate value are shown. Lasing occurs at the maximum of the gain, which is $\lambda=555.5 \mathrm{~nm}$. The width of the lasing peak is limited by the losses through the sample boundaries and by the gain saturation, and, as we confirmed, it is not affected by the numerical spectral
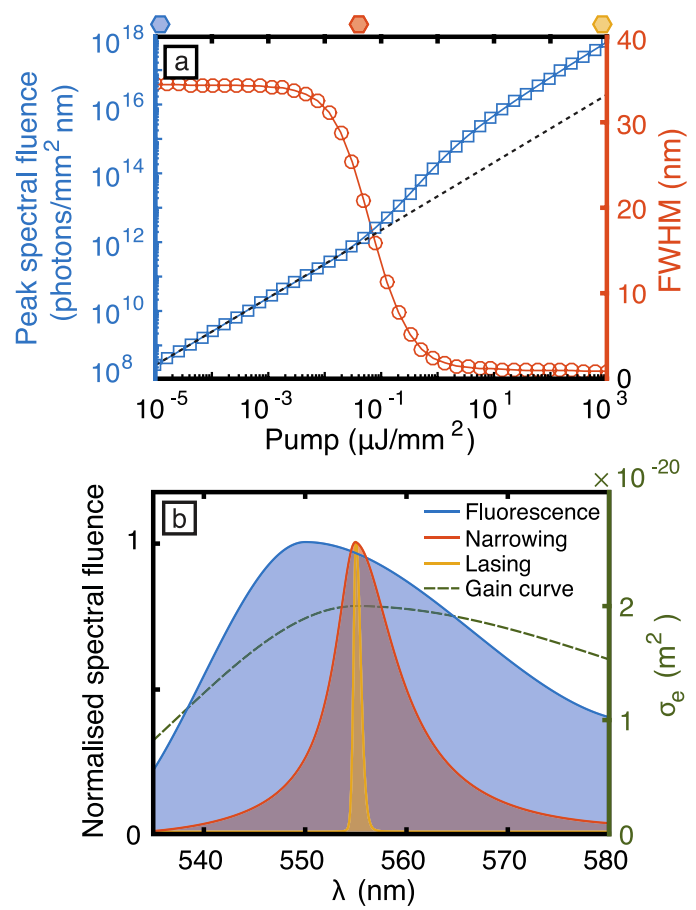

Fig. 1. (a) Characteristic plot of a random laser: the total peak intensity (blue squares) and FWHM of the emission spectrum (red circles) are plotted for increasing pump energy density for a slab system of thickness $L=50 \mu \mathrm{m}$, transport scattering length $\ell_{t}=1.5 \mu \mathrm{m}$, and a $1 \mathrm{mM}$ concentration of rhodamine $6 \mathrm{G}$ providing the gain, excited by a single pulse of duration $d=6 \mathrm{~ns}$ at $532 \mathrm{~nm}$. The black dashed line is a guide to the eye to highlight the change of regime from fluorescence to lasing. (b) The emission spectra (normalised) are reported at different pump intensities: the fluorescence spectrum (blue) narrows down for increasing pump intensity (red line) down to a $0.8 \mathrm{~nm}$ FWHM peak (yellow line). The corresponding pump intensities are colour coded by the hexagons in panel (a). The lasing occurs at the maximum of the gain curve (dashed green line).

discretisation which is set to $0.1 \mathrm{~nm}$. This value differs from the typical experimental values where the final linewidth is typically in the range 5 to $10 \mathrm{~nm}[6,26]$, as we are not considering any additional homogeneous and inhomogeneous line-broadening effects.

We consider now the case of a medium characterised by resonant Mie scattering. We simulate a system composed of close-packed $(n=1.6$, filling fraction $f=0.5)$ dielectric spheres with $1 \%$ radial polydispersity. We calculate $\ell_{t}$ by using Mie theory in the approximation of independent scatterers [27]. The Mie resonances modulate $\ell_{t}$ as shown by the full lines in Fig. 2(a), where scattering is plotted together with the predicted lasing peaks for three sphere diameters. Qualitatively, the lasing frequency is pulled towards the strongest scattering frequency within the gain curve. Compared to what is shown in Fig. 1(b), now the lasing frequency can be tuned by choosing the Mie resonance. 


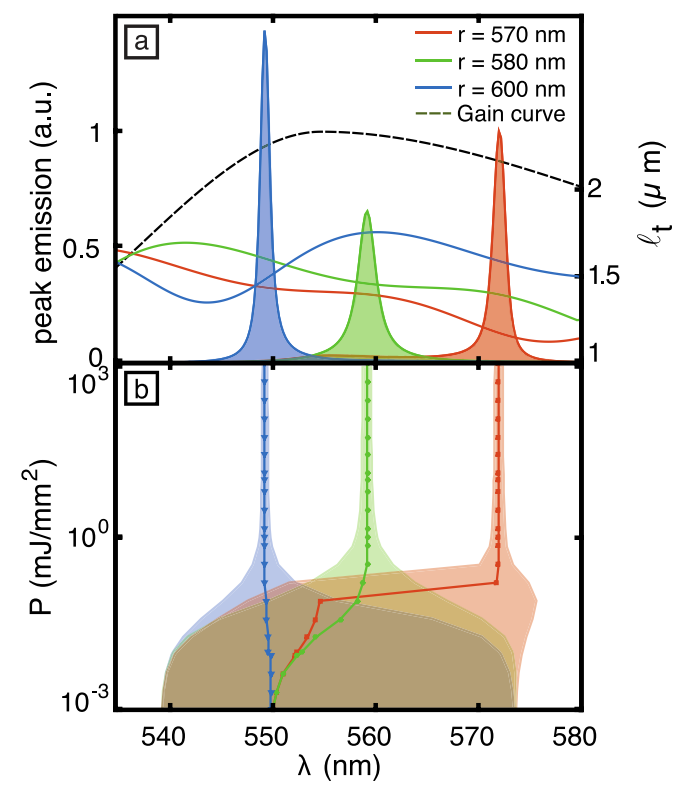

Fig. 2. The effects of resonant scattering on the lasing spectrum. (a) The Mie resonances of spherical scatterers $(n=1.6$ in air) of similar size (polydispersty 1\%) are exploited to tune the lasing emission. The emission wavelength is close to the minimum of the transport mean free path while inside the gain region (black line). (b) At increasing pump power the emission peak wavelength shifts from the fluorescence maximum to the lasing wavelength stabilizing above threshold.

Fig. 2(b) shows the narrowing and shifting of the emission peak while increasing the pump energy. The crossing of the lasing threshold is now evident as a narrowing of the emission spectrum while the lasing peak shifts away from the maximum of the fluorescence curve. Frequencies close to the maximum of the gain initially dominate, as they receive a larger fraction of the spontaneous emission, but those with larger scattering and gain eventually prevail above threshold.

Finally, we study the range of tunability that can be achieved by resonant scattering. In Fig. 3(a) we present the calculated transport mean free path at $\lambda_{\text {gain }}=$ $555.5 \mathrm{~nm}$, which is the wavelength corresponding to the maximum of the gain. While for very small scatterers, which are in the Rayleigh regime, the scattering increases with the particle size, approaching the Mie regime this trend is reversed. The minimum scattering length is achieved at $r \lesssim \lambda / 2$, which corresponds to the onset of the first Mie resonances, and subsequently $\ell_{t}$ increases with a resonant behaviour, while the resonances get closer and closer. As previously shown in Fig. 2, this modulation in the scattering induces a tuning of the emission wavelength which is now explicit in Fig. 3(b) where we plot the lasing frequency versus the particle reduced radius $r / \lambda_{\text {gain }}$. While in the Rayleigh regime the lasing emission follows the maximum of the gain, for $r>\lambda / 2$ the emission starts to red-shift follow-

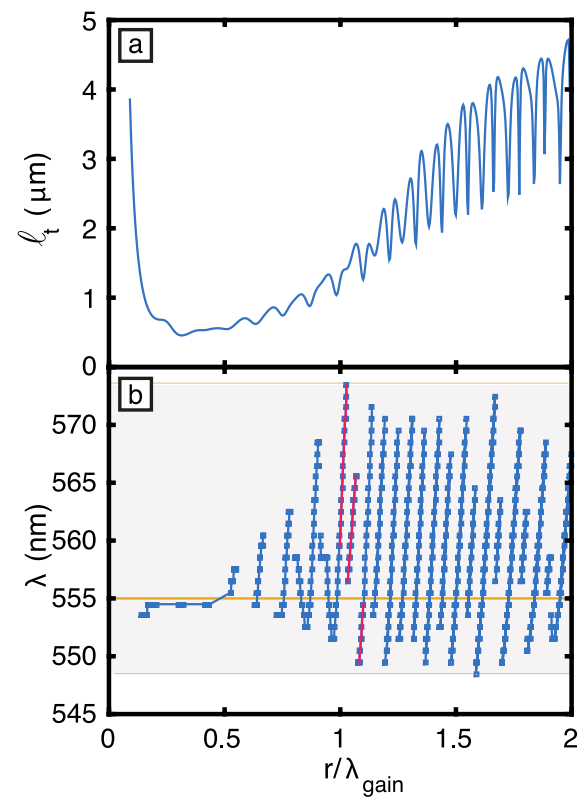

Fig. 3. Tuning the lasing emission wavelength via resonant scattering. (a) Transport scattering mean free path $\ell_{t}$ computed by Mie scattering (spheres of refractive index $n=1.6$ in air, filling ratio $f=0.5$ and $1 \%$ polydispersity) at $\lambda_{\max }=555.5 \mathrm{~nm}$. (b) Lasing emission wavelength for resonant scattering. When the particle size is increased from non-resonant Rayleigh scattering $(r<<\lambda)$ to Mie scattering, the lasing emission wavelength is tuned to follow the scattering resonance in a range of $25 \mathrm{~nm}$ inside the gain curve (gray area). The red lines are further plotted in Fig. 4(a).

ing the Mie resonances. The wavelength jumps correspond to the appearance of a more favorable resonance which pulls the lasing wavelength in the blue part of the gain region. The tunability range for the simulated system is highlighted by the area in grey and it is roughly $25 \mathrm{~nm}$.

Fig. 4(a) is a zoom in of Fig. 3(b) and shows in detail the lasing frequency evolution around $r=\lambda$. The curve consists of three different continuos branches separated by two jumps. The numerical results of the model are compared to analytical diffusion calculations which predict lasing occurring at the wavelength which minimises the critical length $L_{c r}[6,7]$, the length above which the gain exceeds the losses. In a slab geometry, and under the approximation of uniform gain, the critical length is $L_{c r}=\pi \sqrt{\ell_{t} l_{g} / 3}$, where $l_{g}=\left(N \sigma_{e}\right)^{-1}$. The minimum of $L_{c r}$ and the numerical results are in good agreement, with significant differences only close to the wavelength jumps where the simplified analytical model fails to account for the competition for the gain. We attribute this to the increased mode competition between two different Mie resonances. Mode competition is strong for particle sizes around $r=571 \mathrm{~nm}$ and $595.5 \mathrm{~nm}$, and this is reflected by an increased threshold. For instance, the threshold does not always decreases monotonically 


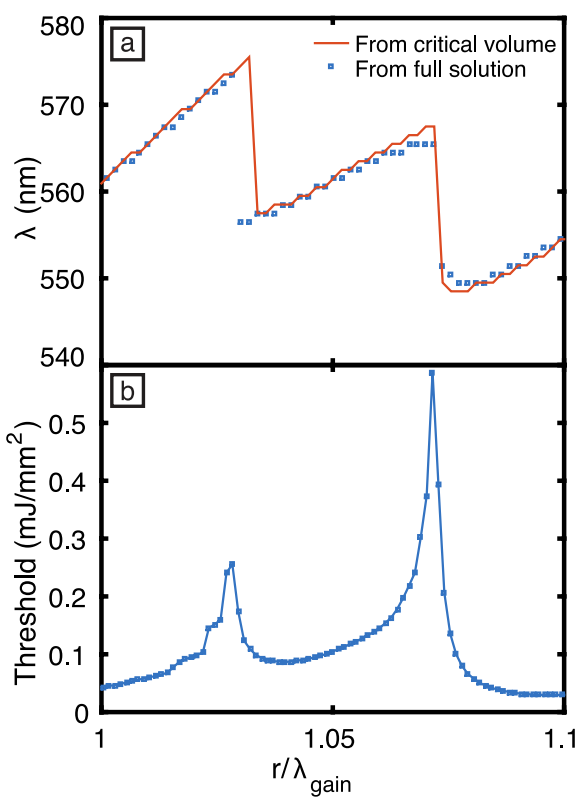

Fig. 4. Details of the emission wavelength and lasing threshold. (a) Pulled by the Mie resonances, the lasing emission redshifts until the next Mie resonance enters the gain spectral region. This competition is shown by the tooth-saw profile. The numerical values (squares) are compared to the analytical prediction (orange line) obtained by the minimum of the critical length $L_{c r}=\pi \sqrt{\ell_{t} \ell_{g} / 3}$. (b) The threshold, defined as the point of half emission narrowing, is quite constant in the tuning range but close to the frequency jumps, when the competition between the two lasing peaks strongly increases it.

when the lasing emission approaches $\lambda_{\text {gain }}$. For the other lasing frequencies the threshold is quite constant in the whole region of tunability with values around $0.1 \mathrm{~mJ} / \mathrm{mm}^{2}$. In addition, we confirm (not shown here) that for particles size $r=571.9 \mathrm{~nm}$ and $r=595.4 \mathrm{~nm}$ the spectrum develops two competing peaks of which one finally prevails for large enough pump energy.

In conclusion, a dispersive diffusive gain model is capable to simulate RL action when driven by gain or scattering resonances. For resonant scattering we calculated a $25 \mathrm{~nm}$ emission tuning range, with minimal threshold increase. Our dispersive model does not require any assumption about the $\beta$ parameter and can predict mode competition and a non-trivial threshold dependence. As a further extension, the model can include arbitrary absorption curves and dispersion also for the energy velocity, and can be extended to the full three-dimensional case at the expense of increased computing time.

We thank Soraya Caixeiro for fruitful discussions. This research was funded by the Engineering and Physical Sciences Research Council (EPSRC), a Leverhulme Trust Research Grant and a FP7 European project. The data is publicly available in Figshare [28], the code is available on request.

\section{References}

[1] D. Wiersma, Nat. Phys. 4 (2008).

[2] H. Cao, Journal of Physics A: Mathematical and General 39, 467 (2006).

[3] N. Ghofraniha, I. Viola, F. Di Maria, G. Barbarella, G. Gigli, L. Leuzzi, and C. Conti, Nat Commun (2015).

[4] B. Redding, M. A. Choma, and H. Cao, Nat Phot. 6, 355 (2012).

[5] S. Caixeiro, M. Gaio, Marelli B., F. G. Omenetto, and R. Sapienza, In pubblication (2015).

[6] S. Gottardo, R. Sapienza, P. D. Garcia, A. Blanco, D. S. Wiersma, and C. Lopez, Nat Phot. 2, 429 (2008).

[7] R. Uppu and S. Mujumdar, Opt. Express 19, 23523 (2011).

[8] R. G. El-Dardiry and A. Lagendijk, Applied Physics Letters 98, 161106 (2011).

[9] N. Bachelard, J. Andreasen, S. Gigan, and P. Sebbah, Phys. Rev. Lett. 109, 033903 (2012).

[10] T. Hisch, M. Liertzer, D. Pogany, F. Mintert, and S. Rotter, Phys. Rev. Lett. 111, 023902 (2013).

[11] N. Bachelard, S. Gigan, X. Noblin, and P. Sebbah, Nat. Phys. 10, 426 (2014).

[12] S. John and G. Pang, Phys. Rev. A 54, 3642 (1996).

[13] D. S. Wiersma and A. Lagendijk, Phys. Rev. E 54, 4256 (1996).

[14] R. G. S. El-Dardiry, R. Mooiweer, and A. Lagendijk, New Journal of Physics 14, 113031 (2012).

[15] R. M. Balachandran, N. M. Lawandy, and J. A. Moon, Opt. Lett. 22, 319 (1997).

[16] G. A. Berger, M. Kempe, and A. Z. Genack, Phys. Rev. E 56, 6118 (1997).

[17] S. Mujumdar, M. Ricci, R. Torre, and D. S. Wiersma, Phys. Rev. Lett. 93, 053903 (2004).

[18] R. Pierrat and R. Carminati, Phys. Rev. A 76, 023821 (2007).

[19] H. E. Türeci, A. D. Stone, and B. Collier, Phys. Rev. A 74, 043822 (2006).

[20] H. E. Tureci, A. D. Stone, L. Ge, S. Rotter, and R. J. Tandy, Nonlinearity 22, C1 (2009).

[21] X. Jiang and C. M. Soukoulis, Phys. Rev. Lett. 85, 70 (2000).

[22] P. Sebbah and C. Vanneste, Phys. Rev. B 66, 144202 (2002).

[23] C. Conti, L. Angelani, and G. Ruocco, Phys. Rev. A 75, 033812 (2007).

[24] R. Sapienza, P. D. García, J. Bertolotti, M. D. Martín, Á. Blanco, L. Viña, C. López, and D. S. Wiersma, Phys. Rev. Lett. 99, 233902 (2007).

[25] W. Holzer, H. Gratz, T. Schmitt, A. Penzkofer, A. Costela, I. Garca-Moreno, R. Sastre, and F. Duarte, Chemical Physics 256, 125 (2000-05-15T00:00:00).

[26] G. van Soest, M. Tomita, and A. Lagendijk, Opt. Lett. 24, 306 (1999).

[27] C. F. Bohren and D. Huffman, Absorption and scattering of light by small particles, Wiley science paperback series (Wiley, 1983).

[28] M. Gaio, M. Peruzzo, and R. Sapienza, doi: 10.6084/m9.figshare.1301143 (2015). 